\title{
Bacteriological Profile of Sepsis Outbreak in the NICU of a Tertiary Care Hospital in Western Nepal
}

\author{
Malla KK ${ }^{1}$, Malla $\mathrm{T}^{2}$, Rao $\mathrm{KS}^{3}$ \\ ${ }^{1}$ Dr. Kalpana K Malla, MD, Professor, ${ }^{2} \mathrm{Dr}$. Tejesh \\ Malla, MD, Associate Professor, ${ }^{3}$ Dr. K. Seshagiri Rao, \\ Professor and HOD Paediatrics. All from the Department \\ of Paediatrics Manipal College of Medical Sciences, \\ Pokhara, Nepal.
}

\section{Introduction}

$\mathrm{N}^{\mathrm{cota}}$ eonatal septicemia remains one of the most important causes of mortality despite considerable progress in hygiene, introduction of new antimicrobial agents, and advanced measures for early diagnosis and treatment ${ }^{1,2}$. Several studies ${ }^{3,4}$ including $\mathrm{Nepal}^{5}$ have observed outbreak of sepsis in their Neonatal Intensive Care Unit (NICU) but the microbials and sensitivity pattern in each varies. Therefore to overcome this problem active surveillance of sepsis and antimicrobial sensitivity of responsible micro-organisms is mandatory in defining the empiric antibiotic regimens. This is the largest tertiary care hospital in Western region of Nepal, and serves as a referral center for the population of this region. Hence a heavy nursing workload is there during overloaded admissions which are a major risk factor for sepsis, especially Nosocomial infection (NI). We describe a scenario of such an outbreak.

\section{Materials and Methods}

This was a prospective observational study conducted in the NICU unit of MTH, Pokhara. The unit has 22 beds, and its occupancy varies from 12 to 22 throughout the year.The study period was from $1^{\text {st }}$ April $2011-15^{\text {th }}$ August 2011. Ethical approval and informed consent from parents were obtained before starting the study. The inclusion criteria for sepsis was positive Creactive protein plus presence of one or more clinical signs consistent with sepsis, lethargy, refusal of feeds, abdominal distension, vomiting, grunting, respiratory distress, hypothermia, hyperthermia or sclerema, seizures, apnea, color changes in skin, petechia with or without supporting evidence of risk factors such as

\footnotetext{
Address for correspondence

Dr. Kalpana Malla

A-7, Staff quarters,

Manipal Teaching Hospital

Pokhara, nepal

E-mail: kalpana01malla@gmail.com
}

\begin{abstract}
Introduction: Neonatal sepsis is a serious problem for the neonates who are admitted to the intensive care and outbreak of sepsis is not uncommon. This paper aims to describe a sepsis outbreak as a result of too many admissions, overcrowding of babies with limited working staffs in the unit and compares microorganisms with their antibiogram in newborn and environmental samples. Materials and Methods: Prospective observational study from $1^{\text {st }}$ April $-15^{\text {th }}$ August 2011 in Neonatal Intensive Care unit of Manipal Teaching Hospital, Pokhara and included all babies admitted for sepsis. Results: There were $103(57.22 \%)$ episodes of neonatal septicemia $($ Term $=47.8 \%$; Preterm $=84.8 \% \mathrm{p}<0.001)$, $14 / 20,70 \%$ of whom died of sepsis. $47.52 \%$ had early onset sepsis and $52.42 \%$ had late onset sepsis and $39.8 \%$ had nosocomial infection. The predominant isolates in newborn (NB) were E. coli, staphylococcus aureus and Klebsiella pneumonia and in environmental sample it was Klebsiella pneumonia and Staphylococcus. aureus. Imipenem, Vancomycin, Netilmycin, Tobramycin and chloramphenicol were sensitive (S-100\%) while Carbenicillin and Piperacillin, Ampicillin, most cephalosporins, Penicillin were resistant (R-100\%) to organisms in newborn and environmental samples. Other S-100\% antibiotics for newborn were Ceftazidime, Ciprofloxacin and Gentamycin while S-100\% for environmental sample isolates were Cephoperazone, Cloxacillin, Cefuroxime and Tetracycline. Other $(R-100 \%)$ antibiotics for newborn were Amoxicillin and Amoxyclav and for environmental sample were Gentamycin and Erythromycin. Conclusions: Sepsis is a severe problem for neonates. Periodic evaluation of bacterial antibiotic susceptibility and judicious selection of antibiotics is necessary to reduce the resurgence of multidrug resistant strains.
\end{abstract}

Key words: Newborn, Neonatal sepsis, Sepsis outbreak

\section{How to cite this article?}

Malla KK, Malla T, Rao KS. Bacteriological Profile of Sepsis Outbreak in the NICU of a Tertiary Care Hospital in Western Nepal. J Nepal Paediatr Soc 2013;33(1):8-14. 
prematurity, low birth weight, birth asphyxia, maternal chorioamnionitis (maternal fever and/or foul smelling vaginal discharge) and prolonged rupture of membranes (>18hrs). Culture positive cases were the gold standard for diagnosis but in conditions where culture was negative other indirect screening parameters 6 like leukopenia - $<5000 / \mathrm{mm} 3$, leukocytosis - >20,000/ $\mathrm{mm} 3$, absolute neutropenia $<1,000$, immature/total neutrophil ratio $>0.2$ were also considered. Patients with respiratory distress syndrome (RDS), gross congenital anomalies, suspected intrauterine infections like Toxoplasmosis Rubella, Cytomegalovirus, Herpes simplex virus infections were excluded. Study patients were categorized as having early onset (72 hours of life) or late onset ( $>72 \mathrm{hrs}-28$ days of life) septicemia. NI was considered when features of sepsis with positive CRP were noted 48 hours after admission. The environmental sample which is routinely sent for cultures was also taken under consideration. The isolated organisms in newborn (NB) and environmental sample (ES) with antimicrobial susceptibility were analyzed. Epi Info version 3.5.2 was used and test applied was F-Test and Chi square test for data analysis. A $p$ value $<.05$ was considered statistically significant.

\section{Results}

All together $103 / 180(57.22 \%)$ episodes of neonatal sepsis [Term $=47.8 \%$; Preterm $=84.8 \% \quad p<0.001$ ] was noted. The mean weight and gestation age was statistically high in term babies (Figure 1). Evidences of sepsis (Early onset sepsis $41.3 \%$ Vs $22.4 \%(p<0.012)$, Late onset sepsis $=52.2 \%$ Vs $22.4 \%(P<0.001)$ and $\mathrm{NI}=30.43 \% \mathrm{Vs} \quad 5.5 .22 \%(\mathrm{p}<0.001) \quad$ were significantly more in preterm. Figure 2 shows distribution of sepsis. In the (NB) samples 27/103 (26.21\%) showed culture positives and 38 (ES) revealed 53/139\% positive isolates, Table 1 Similar growth was noticed in NB and ES during this period.(Table 1). The mortality due to sepsis was $70 \%$ (Figure 3 ). Other causes of mortality were meconium aspiration, perinatal asphyxia,gross congenital anomalies, complex cyanotic congenital heart disease. Table $2 \& 3$ shows the antibiogram of microorganims in NB and ES. The NICU was fully loaded during the outbreak giving nurse-to-patient ratio of $1: 10$ to $1: 11$ in each shift (20 to 22 NB and 4 nurses in morning shift, 3 in afternoon and 3 in night shift.There were two registered nurse and rest volunteers (with less experience and no training), rostered on duties.

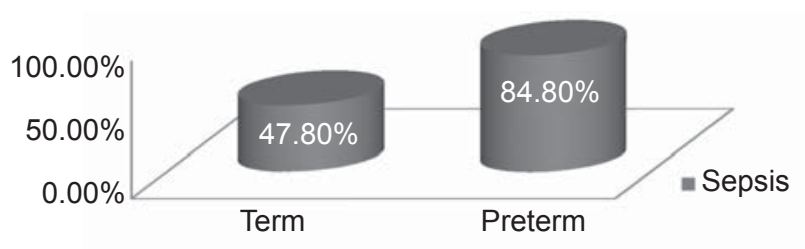

Fig 1: Weight distribution and Sepsis in different gestation age in study population

\begin{tabular}{|c|c|c|c|c|}
\hline & Term $(\mathbf{n}=\mathbf{6 4})$ & Preterm(n=39) & F statistics & p value \\
\hline Mean weight \pm SD & $2723.75 \pm 540.29$ & $1507.94 \pm 392.44$ & 149.23 & 0.001 \\
\hline Mean gestation age \pm SD & $39.29 \pm 1.342$ & $32.00 \pm 2.149$ & 706.32 & 0.001 \\
\hline Sepsis (103) & $64 / 134(47.76 \%)$ & $39 / 46(84.8 \%)$ & & 0.001 \\
\hline
\end{tabular}

Table 1: Organisms isolated in babies and environmental samples

\begin{tabular}{|l|c|c|}
\hline \multirow{2}{*}{ Isolates } & $\begin{array}{c}\text { Newborn samples } \\
\text { Blood, } \\
\text { Cerebrospinal fluid, } \\
\text { Urine }\end{array}$ & $\begin{array}{c}\text { Wt.M, RW, P units, P room, - Breast feeding room, } \\
\text { Air settle plate SC, I,V, Stethoscope, 02 headbox, } \\
\text { Bedside locker, ST }\end{array}$ \\
\hline Klebsiella pneumonia & $5(18.50 \%)$ & $12(22.64 \%)$ \\
\hline Escherichia coli & $10(37 \%)$ & $3(5.66 \%)$ \\
\hline Staphylococcus aureus & $5(18.5 \%)$ & $10(18.86 \%)$ \\
\hline Acinetobacter baumannii & $2(7.40 \%)$ & $4(7.54 \%)$ \\
\hline Coagulase negative staphylococcus & $3(11.11 \%)$ & $7(13.20 \%)$ \\
\hline Enterococcus faecalis & $2(7.40 \%)$ & $8(15.09 \%)$ \\
\hline Pseudomonas aeruginosa & $0(0 \%)$ & $6(11.32 \%)$ \\
\hline Citrobacterfreundii & $0(0 \%)$ & $2(3.77 \%)$ \\
\hline Non-fermenting gram +ve bacilli & $0(0 \%)$ & $\mathbf{1}(1.88 \%)$ \\
\hline \multicolumn{1}{|c|}{ Total } & $\mathbf{n = 2 7}$ & $\mathbf{5 3}$ \\
\hline
\end{tabular}

**Wt.M= Weighing machine, RW= Radiant warmer, P unit = Phototherapy unit, P room = Phototherapy room, SC= Suction catheter, I= Incubator, V= ventilator, $\mathrm{ST}=$ Suction tip 
Table 2: Antibiogram in NB $(n=27)$ and ES $(n=53)$ Percentage sensitive for isolated organisms

\begin{tabular}{|c|c|c|c|c|c|c|c|c|}
\hline \multirow{2}{*}{ Antibiotic } & \multicolumn{2}{|c|}{ KIb } & \multicolumn{2}{|c|}{ CoNS } & \multicolumn{2}{|c|}{ S.aureus } & \multicolumn{2}{|c|}{ E. coli } \\
\hline & NB $(n=5)$ & ES (n=12) & NB $(n=3)$ & ES $(n=7)$ & NB $(n=5)$ & ES $(n=10)$ & NB $(n=10)$ & ES $(n=3)$ \\
\hline Ampicillin & $\begin{array}{l}\mathrm{ST}=4 / 5 \\
\mathrm{~S}=0 \%\end{array}$ & $\begin{array}{c}\mathrm{ST}=9 / 12 \\
\mathrm{~S}=0 \%\end{array}$ & $(-)$ & $\begin{array}{l}\mathrm{ST}=7 / 7 \\
\mathrm{~S}=0 \%\end{array}$ & $(-)$ & $(-)$ & $\begin{array}{c}\mathrm{ST}=8 / 10 \\
\mathrm{~S}=0 \%\end{array}$ & $\begin{array}{c}\mathrm{ST}=3 / 3 \\
\mathrm{~S}=0 \%\end{array}$ \\
\hline Amoxicillin & $\begin{array}{c}\mathrm{ST}=4 / 5 \\
\mathrm{~S}=0 \%\end{array}$ & $\begin{array}{c}\mathrm{ST}=9 / 12 \\
\mathrm{~S}=0 \%\end{array}$ & $(-)$ & $\begin{array}{l}\mathrm{ST}=7 / 7 \\
\mathrm{~S}=0 \%\end{array}$ & $(-)$ & $(-)$ & $(-)$ & $\begin{array}{c}\mathrm{ST}=3 / 3 \\
\mathrm{~S}=0 \%\end{array}$ \\
\hline Amoxyclav & $\begin{array}{c}S T=5 / 5 \\
S=0 \%\end{array}$ & $\begin{array}{c}\mathrm{ST}=9 / 12 \\
\mathrm{~S}=0 \%\end{array}$ & $(-)$ & $\begin{array}{l}\mathrm{ST}=7 / 7 \\
\mathrm{~S}=0 \%\end{array}$ & $(-)$ & $(-)$ & $\begin{array}{l}\text { ST }=6 / 10 \\
1 / 16.66 \%\end{array}$ & $\begin{array}{c}S T=3 / 3 \\
S=0 \%\end{array}$ \\
\hline Ciprofloxacin & $\begin{array}{c}\mathrm{ST}=4 / 5 \\
\mathrm{~S}=2 / 50 \%\end{array}$ & $\begin{array}{c}\mathrm{ST}=12 / 12 \\
\mathrm{~S}=8 / 66.6 \%\end{array}$ & $\begin{array}{l}\mathrm{ST}=3 / 3 \\
3 / 100 \%\end{array}$ & $\begin{array}{c}\mathrm{ST}=7 / 7 \\
\mathrm{~S}=7 / 100 \%\end{array}$ & $\begin{array}{l}\mathrm{ST}=4 / 5 \\
2 / 50 \%\end{array}$ & $\begin{array}{c}\mathrm{ST}=10 / 10 \\
2 / 20 \%\end{array}$ & $\begin{array}{c}S T=10 / 10 \\
10 / 100 \%\end{array}$ & $\begin{array}{c}S T=3 / 3 \\
2 / 66.66 \%\end{array}$ \\
\hline Amikacin & $\begin{array}{c}\mathrm{ST}=5 / 5 \\
\mathrm{~S}=4 / 80 \%\end{array}$ & $\begin{array}{c}\mathrm{ST}=12 / 12 \\
6 / 50 \%\end{array}$ & $(-)$ & $\begin{array}{c}\mathrm{ST}=7 / 7 \\
5 / 71.42 \%\end{array}$ & $(-)$ & $\begin{array}{c}\mathrm{ST}=10 / 10 \\
3 / 30 \%\end{array}$ & $\begin{array}{c}S T=10 / 10 \\
5 / 50 \%\end{array}$ & $(-)$ \\
\hline Gentamycin & $\begin{array}{c}S T=5 / 5 \\
S=1 / 20 \%\end{array}$ & $\begin{array}{l}\mathrm{ST}=12 / 12 \\
8 / 66.66 \%\end{array}$ & $\begin{array}{l}\mathrm{ST}=3 / 3 \\
3 / 100 \%\end{array}$ & $\begin{array}{c}\mathrm{ST}=7 / 7 \\
5 / 71.42 \%\end{array}$ & $\begin{array}{l}\mathrm{ST}=4 / 5 \\
4 / 80 \%\end{array}$ & $\begin{array}{c}\mathrm{ST}=10 / 10 \\
9 / 90 \%\end{array}$ & $\begin{array}{c}S T=10 / 10 \\
5 / 50 \%\end{array}$ & $\begin{array}{c}\mathrm{ST}=3 / 3 \\
\mathrm{~S}=0 \%\end{array}$ \\
\hline Netilmycin & $\begin{array}{c}\mathrm{ST}=5 / 5 \\
\mathrm{~S}=4 / 80 \%\end{array}$ & $\begin{array}{l}\mathrm{ST}=12 / 12 \\
11 / 91.66 \%\end{array}$ & $\begin{array}{l}\mathrm{ST}=2 / 3 \\
2 / 100 \%\end{array}$ & $(-)$ & $(-)$ & $(-)$ & $\begin{array}{l}\mathrm{ST}=8 / 10 \\
7 / 87.5 \%\end{array}$ & $\begin{array}{l}\mathrm{ST}=3 / 3 \\
3 / 100 \%\end{array}$ \\
\hline Tobramycin & $\begin{array}{c}S T=5 / 5 \\
S=4 / 80 \%\end{array}$ & $\begin{array}{c}\mathrm{ST}=12 / 12 \\
\mathrm{~S}=1 / 8.33 \%\end{array}$ & $\begin{array}{c}S T=1 / 3 \\
S=1 / 100 \%\end{array}$ & $(-)$ & $(-)$ & $\begin{array}{c}\mathrm{ST}=10 / 10 \\
7 / 70 \%\end{array}$ & $(-)$ & $(-)$ \\
\hline Chloramphenicol & $\begin{array}{c}S T=5 / 5 \\
S=5 / 100 \%\end{array}$ & $\begin{array}{c}S T=11 / 12 \\
S=5 / 45.45 \%\end{array}$ & $(-)$ & $(-)$ & $(-)$ & $\begin{array}{l}\mathrm{ST}=9 / 10 \\
9 / 100 \%\end{array}$ & $\begin{array}{c}\mathrm{ST}=6 / 10 \\
3 / 50 \%\end{array}$ & $(-)$ \\
\hline $\begin{array}{l}\text { Cefotaxime, } \\
\text { Cephalexin }\end{array}$ & $\begin{array}{c}\mathrm{ST}=4 / 5 \\
\mathrm{~S}=0 \%\end{array}$ & $\begin{array}{c}\mathrm{ST}=10 / 12 \\
\mathrm{~S}=0 \%\end{array}$ & $\begin{array}{c}\mathrm{ST}=1 / 3 \\
\mathrm{~S}=0 \%\end{array}$ & $\begin{array}{l}\mathrm{ST}=2 / 7 \\
\mathrm{~S}=0 \%\end{array}$ & $(-)$ & $\begin{aligned} S T & =6 / 10 \\
S & =0 \%\end{aligned}$ & $\begin{aligned} \mathrm{ST} & =7 / 10 \\
\mathrm{~S} & =0 \%\end{aligned}$ & $\begin{array}{l}\mathrm{ST}=2 / 3 \\
\mathrm{~S}=0 \%\end{array}$ \\
\hline $\begin{array}{l}\text { Ceftriaxone } \\
\text { Cefazoline }\end{array}$ & $\begin{array}{c}\mathrm{ST}=4 / 5 \\
\mathrm{~S}=0 \% \\
\mathrm{ST}=5 / 5 \\
\mathrm{~S}=0 \%\end{array}$ & $\begin{array}{c}\mathrm{ST}=10 / 12 \\
\mathrm{~S}=0 \% \\
(-)\end{array}$ & $\begin{array}{c}(-) \\
S T=3 / 3 \\
S=1 / 33.33 \%\end{array}$ & $\begin{array}{c}\mathrm{ST}=2 / 7 \\
\mathrm{~S}=0 \% \\
(-)\end{array}$ & $\begin{array}{l}\mathrm{ST}=5 / 5 \\
2 / 40 \% \\
\mathrm{ST}=5 / 5 \\
4 / 80 \%\end{array}$ & $\begin{array}{c}\mathrm{ST}=6 / 10 \\
\mathrm{~S}=0 \% \\
\mathrm{ST}=10 / 10 \\
5 / 50 \%\end{array}$ & $\begin{array}{l}\mathrm{ST}=6 / 10 \\
2 / 33.33 \% \\
\mathrm{ST}=6 / 10 \\
1 / 16.66 \%\end{array}$ & $\begin{array}{c}S T=2 / 3 \\
S=0 \% \\
(-)\end{array}$ \\
\hline Cefuroxime & $\begin{array}{l}S T=5 / 5 \\
S=0 \%\end{array}$ & $(-)$ & $(-)$ & $\begin{array}{c}S T=5 / 7 \\
S=5 / 100 \%\end{array}$ & $(-)$ & $(-)$ & $\begin{array}{l}\mathrm{ST}=7 / 10 \\
1 / 14.28 \%\end{array}$ & $\begin{array}{l}S T=2 / 3 \\
S=0 \%\end{array}$ \\
\hline Ceftazidime & $\begin{array}{l}S T=5 / 5 \\
S=0 \%\end{array}$ & $\begin{array}{c}S T=10 / 12 \\
S=\% 0\end{array}$ & $(-)$ & $\begin{array}{c}\mathrm{ST}=2 / 7 \\
\mathrm{~S}=0 \%\end{array}$ & $\begin{array}{c}\mathrm{ST}=4 / 5 \\
1 / 25 \%\end{array}$ & $\begin{aligned} S T & =6 / 10 \\
S & =0 \%\end{aligned}$ & $\begin{array}{l}S T=7 / 10 \\
7 / 100 \%\end{array}$ & $\begin{array}{l}\mathrm{ST}=2 / 3 \\
\mathrm{~S}=0 \%\end{array}$ \\
\hline Cefoperazone & $(-)$ & $(-)$ & $(-)$ & $(-)$ & $(-)$ & $\begin{array}{c}\mathrm{ST}=7 / 10 \\
7 / 100 \%\end{array}$ & $(-)$ & $(-)$ \\
\hline Imipenem- & $\begin{array}{c}S T=4 / 5 \\
S=4 / 100 \%\end{array}$ & $\begin{array}{c}S T=11 / 12 \\
S=11 / 100 \%\end{array}$ & $(-)$ & $(-)$ & $(-)$ & $\begin{array}{l}\mathrm{ST}=8 / 10 \\
8 / 100 \%\end{array}$ & $(-)$ & $(-)$ \\
\hline Cloxacillin & $(-)$ & $(-)$ & $\begin{array}{c}\mathrm{ST}=3 / 3 \\
\mathrm{~S}=2 / 66.66 \%\end{array}$ & $\begin{array}{c}\mathrm{ST}=7 / 7 \\
5 / 71.42 \%\end{array}$ & $\begin{array}{l}\mathrm{ST}=4 / 5 \\
2 / 50 \%\end{array}$ & $\begin{array}{l}\text { ST }=8 / 10 \\
8 / 100 \%\end{array}$ & $(-)$ & $(-)$ \\
\hline Erythromycin & $(-)$ & $(-)$ & $\begin{array}{c}\mathrm{ST}=2 / 3 \\
\mathrm{~S}=0 \%\end{array}$ & $\begin{array}{c}S T=7 / 7 \\
4 / 57.14 \%\end{array}$ & $\begin{array}{l}\mathrm{ST}=5 / 5 \\
3 / 60 \%\end{array}$ & $\begin{array}{c}\mathrm{ST}=5 / 10 \\
\mathrm{~S}=0 \%\end{array}$ & $(-)$ & $(-)$ \\
\hline Carbenecillin & $(-)$ & $\begin{array}{c}\mathrm{ST}=9 / 12 \\
\mathrm{~S}=0 \%\end{array}$ & $(-)$ & $(-)$ & $(-)$ & $\begin{aligned} \mathrm{ST} & =8 / 10 \\
\mathrm{~S} & =0 \%\end{aligned}$ & $(-)$ & $(-)$ \\
\hline Piperacillin & $\begin{array}{l}\mathrm{ST}=5 / 5 \\
\mathrm{~S}=0 \%\end{array}$ & $\begin{array}{c}\mathrm{ST}=10 / 12 \\
\mathrm{~S}=0 \%\end{array}$ & $(-)$ & $(-)$ & $(-)$ & $\begin{aligned} S T & =7 / 10 \\
S & =0 \%\end{aligned}$ & $\begin{aligned} S T & =7 / 10 \\
S & =0 \%\end{aligned}$ & $(-)$ \\
\hline Penicillin & $(-)$ & $(-)$ & $\begin{array}{l}\mathrm{ST}=2 / 3 \\
\mathrm{~S}=0 \%\end{array}$ & $\begin{array}{l}S T=5 / 7 \\
S=0 \%\end{array}$ & $\begin{array}{l}\mathrm{ST}=5 / 5 \\
1 / 20 \%\end{array}$ & $\begin{array}{c}\mathrm{ST}=7 / 10 \\
\mathrm{~S}=0 \%\end{array}$ & $(-)$ & $(-)$ \\
\hline Vancomycin & $(-)$ & $(-)$ & $(-)$ & $(-)$ & $\begin{array}{l}\mathrm{ST}=5 / 5 \\
5 / 100 \%\end{array}$ & $(-)$ & $(-)$ & $(-)$ \\
\hline Tetracycline & $(-)$ & $\begin{array}{c}S T=6 / 12 \\
S=6 / 100 \%\end{array}$ & $(-)$ & $(-)$ & $(-)$ & $(-)$ & $(-)$ & $(-)$ \\
\hline
\end{tabular}

\footnotetext{
**ST=Sensitivity Tested $(-)=$ Sensitivity Not Tested S=sensitive
} 


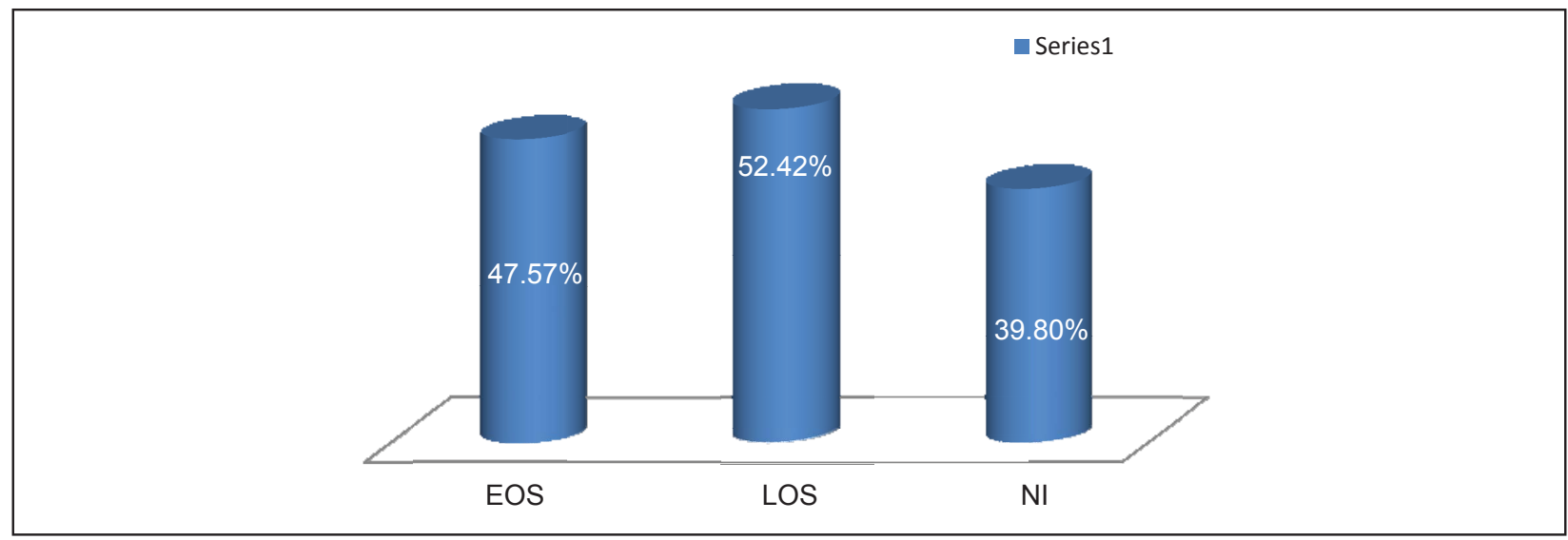

Fig 2: Distribution of Sepsis

Table 3: Antibiogram in NB $(n=27)$ and ES ( $n=53$ - percentage sensitive) for isolated organisms

\begin{tabular}{|c|c|c|c|c|c|c|}
\hline \multirow{2}{*}{ Antibiotic } & \multicolumn{2}{|c|}{ Acinitobacter } & \multicolumn{2}{|c|}{ Enterococcus } & \multirow{2}{*}{$\begin{array}{c}\text { Pseudomonas } \\
\text { ES }(n=6)\end{array}$} & \multirow{2}{*}{$\begin{array}{c}\text { Citrobacter } \\
\text { ES }(n=2)\end{array}$} \\
\hline & NB $(n=2)$ & ES $(n=4)$ & $E S(n=2)$ & $E S(n=8)$ & & \\
\hline Ampicillin & $(-)$ & $(-)$ & $\begin{array}{l}\mathrm{ST}=2 / 2 \\
1 / 50 \%\end{array}$ & $\begin{array}{l}\mathrm{ST}=8 / 8 \\
3 / 37.5 \%\end{array}$ & $\begin{array}{c}\mathrm{ST}=6 / 6 \\
\mathrm{~S}=0 \%\end{array}$ & $\begin{array}{l}\mathrm{ST}=2 / 2 \\
\mathrm{~S}=0 \%\end{array}$ \\
\hline Amoxy \& amoxyclav & $(-)$ & $(-)$ & $(-)$ & $\begin{array}{l}\mathrm{ST}=8 / 8 \\
\mathrm{~S}=0 \%\end{array}$ & $\begin{array}{l}\mathrm{ST}=6 / 6 \\
\mathrm{~S}=0 \%\end{array}$ & $\begin{array}{l}\mathrm{ST}=2 / 2 \\
\mathrm{~S}=0 \%\end{array}$ \\
\hline Ciprofloxacin & $(-)$ & $\begin{array}{l}\mathrm{ST}=3 / 4 \\
\mathrm{~S}=0 \%\end{array}$ & $\begin{array}{l}\mathrm{ST}=2 / 2 \\
1 / 50 \%\end{array}$ & $\begin{array}{l}\mathrm{ST}=8 / 8 \\
6 / 75 \%\end{array}$ & $\begin{array}{c}\mathrm{ST}=6 / 6 \\
4 / 66.66 \%\end{array}$ & $\begin{array}{l}\mathrm{ST}=2 / 2 \\
1 / 50 \%\end{array}$ \\
\hline Amikacin & $(-)$ & $\begin{array}{l}\mathrm{ST}=3 / 4 \\
\mathrm{~S}=0 \%\end{array}$ & $\begin{array}{l}\mathrm{ST}=2 / 2 \\
1 / 50 \%\end{array}$ & $\begin{array}{l}\mathrm{ST}=8 / 8 \\
4 / 50 \%\end{array}$ & $\begin{array}{l}\mathrm{ST}=6 / 6 \\
6 / 100 \%\end{array}$ & $(-)$ \\
\hline Gentamycin & $(-)$ & $\begin{array}{l}\mathrm{ST}=3 / 4 \\
\mathrm{~S}=0 \%\end{array}$ & $\begin{array}{c}\mathrm{ST}=(2 / 2 \\
1 / 50 \%\end{array}$ & $\begin{array}{l}\mathrm{ST}=8 / 8 \\
5 / 62.5 \%\end{array}$ & $\begin{array}{c}\mathrm{ST}=6 / 6 \\
4 / 66.66 \%\end{array}$ & $\begin{array}{l}\mathrm{ST}=2 / 2 \\
\mathrm{~S}=0 \%\end{array}$ \\
\hline Netilmycin & $(-)$ & $(-)$ & $\begin{array}{l}\mathrm{ST}=2 / 2 \\
2 / 100 \%\end{array}$ & $\begin{array}{l}\mathrm{ST}=8 / 8 \\
8 / 100 \%\end{array}$ & $(-)$ & $\begin{array}{l}\mathrm{ST}=2 / 2 \\
2 / 100 \%\end{array}$ \\
\hline Tobramycin & $\begin{array}{l}\mathrm{ST}=0 / 2 \\
\mathrm{~S}=0 \%\end{array}$ & $\begin{array}{l}\mathrm{ST}=2 / 4 \\
\mathrm{~S}=0 \%\end{array}$ & $(-)$ & $(-)$ & $\begin{array}{l}\mathrm{ST}=6 / 6 \\
6 / 100 \%\end{array}$ & $(-)$ \\
\hline Chloramphenicol & $(-)$ & $(-)$ & $(-)$ & $\begin{array}{l}\mathrm{ST}=5 / 8 \\
5 / 100 \%\end{array}$ & $(-)$ & $(-)$ \\
\hline $\begin{array}{l}\text { Taxime, ceftriaxone, } \\
\text { ceftazidime, cephalexin }\end{array}$ & $(-)$ & $\begin{array}{l}\mathrm{ST}=3 / 4 \\
\mathrm{~S}=0 \%\end{array}$ & $\begin{array}{l}\mathrm{ST}=2 / 2 \\
\mathrm{~S}=0 \%\end{array}$ & $\begin{array}{l}\mathrm{ST}=7 / 8 \\
\mathrm{~S}=0 \%\end{array}$ & $\begin{array}{l}\mathrm{ST}=5 / 6 \\
\mathrm{~S}=0 \%\end{array}$ & $\begin{array}{l}\mathrm{ST}=1 / 2 \\
\mathrm{~S}=0 \%\end{array}$ \\
\hline Cefazoline & $(-)$ & $(-)$ & $(-)$ & $\begin{array}{l}\mathrm{ST}=6 / 8 \\
\mathrm{~S}=0 \%\end{array}$ & $(-)$ & $\begin{array}{l}\mathrm{ST}=2 / 2 \\
\mathrm{~S}=0 \%\end{array}$ \\
\hline Cefuroxime & $(-)$ & $(-)$ & $(-)$ & $(-)$ & $(-)$ & $(-)$ \\
\hline Imipenem & $\begin{array}{l}\mathrm{ST}=2 / 2 \\
2 / 100 \%\end{array}$ & $\begin{array}{l}\mathrm{ST}=4 / 4 \\
4 / 100 \%\end{array}$ & $(-)$ & $(-)$ & $(-)$ & $(-)$ \\
\hline Cloxacillin & $(-)$ & $(-)$ & $\begin{array}{c}\mathrm{ST}=2 / 2 \\
1 / 50 \%\end{array}$ & $\begin{array}{c}\mathrm{ST}=7 / 8 \\
\mathrm{~S}=0 \%\end{array}$ & $(-)$ & $(-)$ \\
\hline Erythromycin & $(-)$ & $(-)$ & $\begin{array}{l}\mathrm{ST}=2 / 2 \\
\mathrm{~S}=0 \%\end{array}$ & $\begin{array}{l}\mathrm{ST}=7 / 8 \\
\mathrm{~S}=0 \%\end{array}$ & $\begin{array}{l}S T=2 / 6 \\
S=0 \%\end{array}$ & $(-)$ \\
\hline Carbenecillin & $\begin{array}{l}S T=2 / 2 \\
S=0 \%\end{array}$ & $\begin{array}{l}\mathrm{ST}=3 / 4 \\
\mathrm{~S}=0 \%\end{array}$ & $(-)$ & $(-)$ & $\begin{array}{l}\text { ST=5/6 } \\
5 / 100 \%\end{array}$ & $(-)$ \\
\hline Piperacillin & $\begin{array}{l}\mathrm{ST}=0 / 2 \\
\mathrm{~S}=0 \%\end{array}$ & $\begin{array}{l}\mathrm{ST}=3 / 4 \\
\mathrm{~S}=0 \%\end{array}$ & $\begin{array}{l}\mathrm{ST}=2 / 2 \\
\mathrm{~S}=0 \%\end{array}$ & $\begin{array}{l}\mathrm{ST}=4 / 8 \\
\mathrm{~S}=0 \%\end{array}$ & $\begin{array}{l}\text { ST }=5 / 6 \\
5 / 100 \%\end{array}$ & $\begin{array}{l}\mathrm{ST}=1 / 2 \\
\mathrm{~S}=0 \%\end{array}$ \\
\hline Penicillin & $(-)$ & $(-)$ & $(-)$ & $(-)$ & $(-)$ & $(-)$ \\
\hline Vancomycin & $(-)$ & $(-)$ & $\begin{array}{l}\mathrm{ST}=2 / 2 \\
2 / 100 \%\end{array}$ & $\begin{array}{l}\mathrm{ST}=8 / 8 \\
8 / 100 \%\end{array}$ & $(-)$ & $(-)$ \\
\hline Tetracycline & $(-)$ & $\begin{array}{l}\mathrm{ST}=4 / 4 \\
4 / 100 \%\end{array}$ & $(-)$ & $\begin{array}{l}S T=4 / 8 \\
4 / 100 \%\end{array}$ & $(-)$ & $\begin{array}{l}\mathrm{ST}=2 / 2 \\
2 / 100 \%\end{array}$ \\
\hline
\end{tabular}

**ST=Sensitivity Tested $(-)=$ Sensitivity Not Tested $\mathrm{S}=$ sensitive 


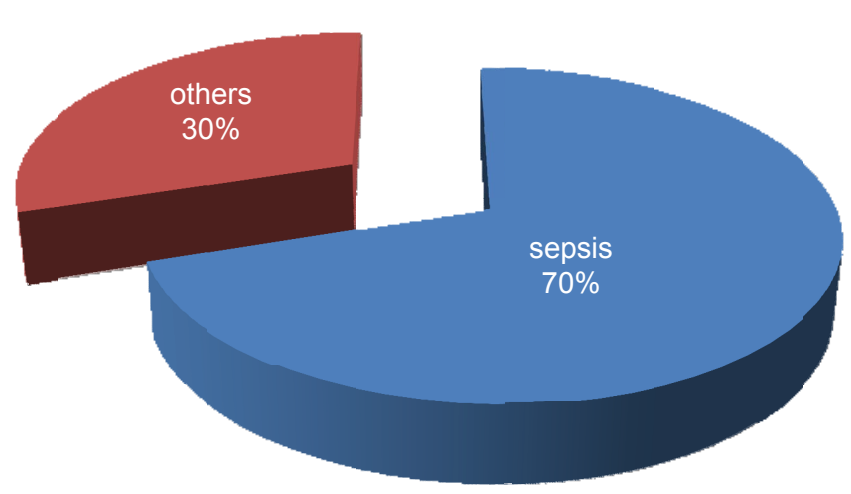

Fig 3: Mortality during study period

\section{Discussion}

The outbreak of sepsis is noticed in almost all NICUs. The presumed source of infection is an infected neonate who subsequently causes cross-infection and colonization of the nursery environment. Despite routine hand washing, minimal handling of newborns and environmental decontamination procedures which is practiced in our NICU, we observed a sepsis outbreak. The possible contributing factors for sepsis outbreak can be organisms continuing to spread between neonates via the contaminated hands of health workers, Cots lined up with no space in between, nursing staffs not routinely washing hands while handling newborns, lack of adequate facilities, understaffing and overcrowding. It is estimated that up to $20 \%$ of neonates develop sepsis and approximately $1 \%$ die of sepsis related causes ${ }^{7}$. During three and half month study period we observed $57.22 \%$ episodes of sepsis. In same institute in year $2007^{5}$, a study over 6 years had shown $49.43 \%$ episodes of neonatal sepsis which is less than this outbreak. Only $9.31 \%$ of sepsis was noted in NICU of Taipei, Taiwan over a period of two years, ${ }^{3}$ this indicates that scenario may vary in different settings. Sepsis was more in PT $(84.8 \%)$ babies. The reason for this is premature babies have low immunity; they need additional supports like ventilation, intravenous fluids, or blood products, and stay in hospital for longer time. Sepsis has got a special challenge for neonatologists and can be very serious leading to very high mortality. In this study also there was $70 \%(14 / 20)$ sepsis deaths. Forty percent (11) and $7.22 \%$ (3) mortality was observed by other authors which is lower than ours. The reason for higher mortality in our study is those patients are referred very late in our hospital when they fail to manage in other centers.

Infections occurring more than 48 hours after admission are usually considered nosocomial ${ }^{8}$. NI occur worldwide, both in the developed and developing world. In this study there was $39.8 \%$ of NI. Patients usually acquire the infection during the procedure itself, either endogenously from flora on the skin or exogenously from air, medical equipment, doctors, or other staff. In other studies $24.6 \%{ }^{9}$ and $45 \%{ }^{4}$ of $\mathrm{NI}$ was detected. Another major issue for $\mathrm{NI}$ is nurse to patient ratio which was $1: 10$ to $1: 11$ in this study. Similar patient to nurse ratio was reported by other authors ${ }^{10}$. In the United States, guidelines recommend a nurse-to-patient ratio of $1: 1$ for patients who are unstable and severely ill, 1:2 for patients who are stable but severely ill, and 1:4 for patients who are stable ${ }^{10}$. In this study there were $21.35 \%$ culture positive sepsis. In another study $35 \%$ and $34.88 \%$ had a positive culture ${ }^{11,12}$. which is similar to our findings ${ }^{15}$. Though the percentage of culture positive cases are similar the isolated organisms differ in different settings. Group B streptococcal (GBS) sepsis is the most important cause of neonatal sepsis in Europe and North America ${ }^{13}$, but there is a preponderance of gram-negative organisms in tropical and developing countries $^{14}$. The epidemiology of neonatal septicemia within a geographical location, however, also may change with time ${ }^{15,16}$. Group B Streptococcus (GBS) was not isolated in this study. The insignificance of GBS as a pathogen in many developing countries is supported by a number of other studies ${ }^{17,18}$. This may be attributable to low prevalence of GBS colonization of pregnant mothers in this area or possibly, to the presence of strains with low virulence. In this study, $E$. coli was the predominant organisms in newborn sample followed by S.aureus and klebsiella while $S$. aureus and Klebsiella was predominant organism isolated in $E S$. The prevalence of $E$. coli may be due to the fact that it is commonly found as part of the intestinal and vaginal flora, and most deliveries are conducted at home, presumably under conditions of poor hygiene. Similar scenario was also observed in an Indian study ${ }^{19}$, but unlike our study they also had high incidence of fungal infection causing sepsis. Yet in another study the $19.2 \%$ of fungal infections was reported ${ }^{20}$. Klebsiella and S.aureus were reported also by other authors ${ }^{21}$. 
The organisms grown in the NB during the outbreak were associated with similar environmental growth 6/9 $(66.66 \%)$. This signifies high incidence of nosocomial infection.

Antibiotic susceptibilities: It is difficult to compare antibiotic susceptibility patterns between countries because the epidemiology of neonatal sepsis is extremely variable. Most of the isolates in this study showed high rates of resistance to almost all cephalosporins both in NB and ES. Only few isolates like CoNS, S. aureus and E.coli were highly sensitive to Cefuroxime, Ceftazidime, Cefoperazone $(\mathrm{R})$ to third generation cephalosporins (> $80 \%$ ) was also observed in another study ${ }^{19}$. They also observed (R-50-75\%) to aminoglycosides which differed from our study. In our study aminoglycosides were highly sensitive. Netilmycin was $\mathrm{S}-100 \%$ for most isolates [CoNS, Enterococcus, S.aureus, E.coli, Pseudomonas and citrobacter], Gentamycin was S-71.42\%- 100\% to CoNS and S.aureus, Amikacin and Tobramycin was S-100\% for pseudomonas and S-50 - 80\% for Klebsiella, Enterococcus, E.coli. We found Acintobacter highly resistant to all antibiotics except imipenem [S-100\% = NB\&ES] and Tetracyclin [S-100\% = ES]. But in other study they were also sensitive to ciprofloxacin (96.2\%), amikacin $(92.4 \%)$ and gentamycin $(87.3 \%)^{22}$. Imipenem was also highly sensitive to $S$. aureus, Klebsella in our study. A 20\% resistance to Imipenem was observed in another study, ${ }^{17}$ but our study showed no resistance to imipenem. A finding similar to ours was noted in another study ${ }^{12}$. Other highly (S) antibiotics in this study were Chloramphenicol [Klebsiella, S. aureus and Enterococcus], Tetracyclin [Klebsiella, Enterobacter, Citrobacter and Acintobacter], Vancomycin [S. aureus, Enterococcus] and Ciprofloxacin [CoNS, E.coli]. In other study bacteria was less resistant (30-46\%) to piperacillintazobactam ${ }^{17}$. But in this study it was highly resistant $(100 \%)$ to maximum isolates [Klebsiella, Acintobacter, Enterococcus $=$ NB and ES, S.aureus, citrobacter and pseudomonas $=\mathrm{ES}]$. Other $100 \%$ resistant antibiotic was Ampicillin [Klebsiella, E.coli, Acintobacter, CoNS, Pseudomonas and citrobacter], Erythromycin [Klebsialla, CoNS, S.aureus, pseudomonas, enterococcus], Amoxicillin, Amoxyclav [Klebsiella], Penicillin [CoNS, S.aureus], Carbenicillin [Klebsiella, S.aureus, Pseudomonas, E.coli \&citrobacter, E.coli, Acinitobacter], Tobramycin [citrobacter], ciprofloxacin [Acinitobacter]. A study done in the same unit 5 years ago also showed resistance to most cephalosporins, penicillins, aminoglycosides and effective antibiotics were imipenem and cefipime ${ }^{5}$.

To control infections, prolonged use of broadspectrum antibiotics is often encountered, which leads to the resurgence of multidrug-resistant organisms. Therefore, preventive antibiotics should be used as little as possible, while therapeutic antibiotics should be specific and used for short period of time. Possible strategies to be considered might include simple infection control methods such as hand washing and barrier nursing, promotion of clean deliveries, exclusive breast feeding, judicious use of antibiotic, and rationalization of admissions to and discharges from neonatal units.

\section{Conclusion}

In conclusion, different NICUs have different epidemiologies of nosocomial infections. Collection of up-to-date data is mandatory for appropriate use of antibiotics, and strategies to avoid the resurgence of multidrug resistant strains should be established. Every unit must follow the bacterial spectrum and antibacterial resistance patterns to choose their specific empirical treatment strategy for sepsis. Due to the small sample size and hospital-based design of this study, we recommend additional community-based studies of local patterns and antibiotic sensitivity of pathogens of neonatal septicemia in order to formulate rational antibiotic use policies.

Acknowledgement: We would like to thank Dr. Prithuja Poudyal for her active participation in data collection. We would also like to thank Dr. Dharm Raj Bhatta, Lecturer, Department of microbiology for helping us with the cultures and sensitivity pattern for different samples.

Funding: None

Competing interests: None

Permission from IRB: Yes

\section{References}

1. Gotoff SP. Neonatal sepsis and meningitis: In: Nelson Textbook of Pediatrics (15th Edition). Eds Behrman RE, Kleigman RM, Arvin AM. Philadelphia, WB Saunders Company, 1996; 528-537.

2. Haque $\mathrm{KH}$. Infection and immunity in the newborn. In: Forfar and Arneil's Textbook of Pediatrics (5 $5^{\text {th }}$ Edition). Eds Campbell AGM, Macintosh N. Pearson Professional Limited,1988;273-289.

3. Ni-Chung Lee, Shu-Jen Chen, Ren-Bin Tang, BeTau Hwang. Neonatal Bacteremia in a Neonatal Intensive Care Unit: Analysis of Causative Organisms and Antimicrobial Susceptibility. J Chin Med 2004;67:15-20.

4. Bhutta ZA, Naqvi SH, Muzzaffar T, Farooqui J. Neonatal sepsis in Pakistan: Presentation and pathogen. Acta Pediatr Scand 1991; 80: 596-601.

5. Shaw CK, Shaw P, Thapalial A. Neonatal sepsis bacterial isolates and antibiotic susceptibility patterns at a NICU in a tertiary care hospital in 
western Nepal: a retrospective analysis. KUMJ 2007; 5(2):153-60.

6. Karen M. Puopolo. Bacterail and Fungal Infections:In:Manual of Neonatal care(6 $6^{\text {th }}$ Edition). Eds Cloherty, John P;Eichenwald, Eric C; Stark, Ann R. Lippincott Williams and Wilkins, 2008;274-300.

7. Stoll BJ. The global impact of neonatal infection. Clin Perinatol 1997;24:1-21.

8. Ducel G, Fabry J, and L. Nicolle. "Prevention of hospital acquired infections: A Practical Guide, $2^{\text {nd }}$ Edition," World Health Organization 2002;4-5.

9. VG. Hemming, MD, James C. Overall. Jr, MD, and Michael R. Britt, M.D. Nosocomial Infections in a Newborn Intensive-Care Unit - Results of FortyOne Months of Surveillance. N Engl J Med 1976; (10); 294:1310-1316.

10. Casolari C, Pecorari M, Fabio G, Cattani S, Venturelli C, Piccinini L, et.al. A simultaneous outbreak of Serratia marcescens and Klebsiella pneumoniae in a neonatal intensive care unit. J Hosp Infect 2005;61:312-320.

11. Tsering DC, Chanchal L, Pal R, Kar S. Bacteriological profile of septicemia and the risk factors in neonates and infants in Sikkim. J Global Infect Dis 2011;3:425 .

12. A.S.M. Nawshad Uddin Ahmed, M.A.K. Azad Chowdhury, Mahbul Hoque and Gary L. Darmstadt. Clinical and Bacteriological Profile of Neonatal Septicemia in a Tertiary level Pediatric Hospital in Bangladesh. Indian Pediatr 2002;39:1034-1039.

13. Fisher G, Horton RE, Edelman R. Summary of the Neonatal Institute of Health Workshop on Group B streptococcal infections. J Infect Disease 1983;148:163-166.
14. Sharma PP, Halder D, Dutta A. Bacteriological profile of neonatal septicemia. Indian Pediatr 1987; $11: 1010-1017$

15. Saha SK, Rikitomi N, Ruhulamin M, Watanabe K, Ahmed K, Biswas D, et al. The increasing burden of disease in Bangladeshi children due to Hemophilus influenzae type b meningitis. Ann Trop Pediatr 1997;17:5-8.

16. Glandstone IM, Ehrenkranz RA, Edberg SC, Baltimore RS. A ten-year review of neonatal sepsis and comparison with the previous fifty year experience. Pediatr Infect Dis J 1990;9:819-825.

17. Walsh JA, Hutchins S. Group B streptococcal disease: its importance in the developing world and prospect for prevention with vaccines. Pediatr Infect Dis J 1989;8:271-276.

18. Duruvilla KA, Thomas N, Jesudasan MV, Jana AK. Neonatal group B streptococcal bacteremia in India: ten years' experience. Acta Pediatr 1999; 88: 27/103 (26.21\%) 1031-1032.

19. David Isaacs, Neonatal Sepsis: The Antibiotic Crisis. Indian Pediatr 2005;42:9-13.

20. Mehmet Yalaz1, Hasan Çetin1, Mete Akisu1, Şöhret Aydemir. Neonatal nosocomial sepsis in a level-III NICU: evaluation of the causative agents and antimicrobial susceptibilities. The Turkish $\mathrm{J}$ of Pediatr. 2006;48:13-18.

21. Karunasekera KA, Pathirana D. A preliminary study on neonatal septicemia in a tertiary referral hospital pediatric unit. Ceylon Med J 1999;44:81-6.

22. Asit Mishra, Sudhir Mishra, Geetha Jaganath, Raj K. Mittal, P.K. Gupta and D.P. Patra. Acintobacter sepsis in newborns. Indian Pediatr 1998;35:27-32. 